\title{
GALERKIN METHODS IN THE CONSTRUCTIVE SOLVABILITY OF NONLINEAR HAMMERSTEIN EQUATIONS WITH APPLICATIONS TO DIFFERENTIAL EQUATIONS
}

\author{
BY
}

P. M. FITZPATRICK ${ }^{1}$ AND W. V. PETRYSHYN ${ }^{2}$

\begin{abstract}
We consider the solution of abstract Hammerstein equations by means of a Galerkin approximating scheme. The convergence of the scheme is proven by first establishing an equivalent scheme in a Hilbert space and then proving a convergence result for firmly monotone operators in a Hilbert space. The general results are applied to the case when the involved linear mapping is angle-bounded, and also to the treatment of certain differential equations.
\end{abstract}

Introduction. If $\Omega$ is a measure space whose measure is denoted by $d y$, a nonlinear integral equation of the form

$$
u(x)+\int_{\Omega} k(x, y) f(y, u(y)) d y=0, \quad x \in \Omega,
$$

is called a Hammerstein integral equation. Typically, $k: \Omega \times \mathbf{R} \rightarrow \mathbf{R}$ would be the Green's function of a differential operator $L$, and obtaining solutions of the above equation would be equivalent to solving the differential equation

$$
L(u)(x)+f(x, u(x))=0,
$$

together with boundary conditions, which would be accounted for in the composition of $k$.

If $X$ and $Y$ denote classes of integrable functions on $\Omega$ such that the operators $K: X \rightarrow Y$ and $N: Y \rightarrow X$, defined by

$$
K(u)(x)=\int_{\Omega} k(x, y) u(y) d y, \quad \text { for } x \in \Omega, u \in X,
$$

and

$$
N(v)(y)=f(y, v(y)), \quad \text { for } y \in \Omega, v \in Y,
$$

Received by the editors August 16, 1976.

AMS (MOS) subject classifications (1970). Primary 45G99, 34B15, 47H05.

Key words and phrases. Integral equations, Hammerstein, Galerkin approximation, firmly monotone operator, differential equation.

${ }^{1}$ Supported in part by NSF Grant MCS 76-07461.

${ }^{2}$ Supported in part by NSF Grant MPS 75-08412.

- American Mathematical Society 1978 
make sense, $(0.1)$ may be rewritten as

$$
u+K N(u)=0, \quad u \in Y .
$$

The latter formulation is preferable in some situations because, first, the various hypotheses imposed on $k$ and $f$ can be put into a conceptually clearer framework, and secondly because it allows one to treat problems which are a good deal more general than (0.1). For instance, it often happens that the operator $L$ in $(0.2)$ is invertible, when one selects a suitable class of generalized functions, and so letting $K=(L)^{-1}$ we see that $(0.2)$ may be reformulated as (0.3), even though no assertion about the existence of a Green's function has been made; moreover, the abstract formulation does not restrict one to nonlinearities of the above type.

In the present paper we are going to seek solutions of $(0.3)$ by means of Galerkin approximations; i.e. we will associate with (0.3) a sequence of finite-dimensional equations, each of which is, in a sense, an approximation of (0.3), and then deduce results about the existence of solutions of (0.3) from the existence of solutions of these approximating equations. Moreover, our results will be constructive in the sense that solutions to the general equation will be obtained as limits to solutions of the finite-dimensional equations. Our approach is similar to Amann's [2], [3] for the case when $X$ is a reflexive Banach space, $K: X \rightarrow X^{*}$ is angle-bounded, $N: X^{*} \rightarrow X$ is monotone and continuous. As will be seen in $\$ 4$ our results extend those of [2], [3] and, in addition, we are able to treat the case when $K: X^{*} \rightarrow X$ and $N: X \rightarrow X^{*}$, with $X$ nonreflexive. We are able to obtain the convergence of the Galerkin method under conditions that are usually associated with existence results and proven by some topological argument. Moreover, the generality of our results is such that, as will be seen in $\$ 5$, various types of nonlinear differential equations can be constructively solved by application of our abstract results.

$\$ 1$ is devoted to establishing the setting in which the equations will be considered. $X$ and $Y$ are real Banach spaces with [, ] a continuous bilinear form on $X \times Y$. The linear operator $K: X \rightarrow Y$ is assumed to be such that it may be factored through a Hilbert space $H$, and then sequences $\left\{X_{n}\right\}$ and $\left\{Y_{n}\right\}$ of finite-dimensional subspaces of $X$ and $Y$, respectively, are defined, leading to the Galerkin equation

$$
[x, y+K N(y)]=0, \text { for all } x \in X_{n}, \text { where } y \in Y_{n} \text {. }
$$

$(0.3)$ is then transformed into an equation in the Hilbert space, and a classical Galerkin scheme for this new equation is shown to be equivalent to the original Galerkin scheme. All of the results are algebraic.

In the second section we prove a general result, Theorem 2.1, concerning the compactness of bounded sequences of solutions of Galerkin equations for 
the equation

$$
T(w)=0, \quad w \in W
$$

where $W$ is a Banach space and $T: W \rightarrow W^{*}$ is a compact perturbation of a strongly monotone mapping; the Galerkin equations are defined on a sequence of subspaces $\left\{W_{n}\right\}$ of $W$ on which we make no assumptions of denseness in $W$. In particular, $W$ is not assumed to be separable, and, as is shown later, this allows one to examine Galerkin schemes for angle-bounded mappings $K$ from $X^{*}$ into $X$ in the situation that $X^{*}$ need not be separable.

$\$ 3$ is concerned with combining the results of the previous two sections in order to obtain general constructive existence results, Theorems 3.1 and 3.2, for $(0.3)$ with a Galerkin scheme defined by $(0.4)$. We prove the existence of solutions to $(0.4)$ which remain bounded, then use the results of $\S 1$ to transform these solutions into solutions of a Galerkin scheme in $H$, apply the results of $\$ 2$ to conclude that the transformed solutions form a relatively compact set, and then again use the results of $\S 1$ to get the relative compactness of the sequence of solutions to (0.4).

The last two sections are devoted to the application of the results of $\$ 3$ to more specific situations. In $\$ 4$ we consider the case, treated in [3] under stronger conditions on $N$, when $K: X \rightarrow X^{*}$ or $K: X^{*} \rightarrow X$ is angle-bounded, and give conditions under which the Galerkin approximating equations may be solved and will have subsequences which converge strongly to solutions of (0.3). A splitting lemma of Browder and Gupta [7] plays an essential part in this discussion. Angle-bounded mappings include as a special subclass positive symmetric mappings, and so our results here extend the classical results for such mappings which have been achieved by the direct methods of the calculus of variations via Ritz-Galerkin schemes (see [6], [8], [10], [11] and the references cited therein).

Lastly we apply the results of $\$ 3$ to two differential equations, the first of which is

$$
\left\{\begin{array}{l}
u^{\prime \prime}(t)+f\left(t, u(t), u^{\prime}(t), u^{\prime \prime}(t)\right)=0, \text { on }(0,1), \\
u(0)=u(1)=0
\end{array}\right.
$$

and the second of which is a partial differential equation whose linear part is the biharmonic operator. Our results in $\$ 3$ are applied to these equations by means of Proposition 5.1, a result which we feel may be of independent interest; it shows how to factor certain bijections through bounded mappings and produces the Hilbert space through which these mappings act.

1. Let $X$ and $Y$ be real Banach spaces, with [, ]: $X \times Y \rightarrow \mathbf{R}$ a continuous bilinear mapping. Suppose $N$ : $\operatorname{Dom}(N) \subseteq Y \rightarrow X$ and $K: \operatorname{Dom}(K) \subseteq X \rightarrow Y$ are given. Then the equation in which we are interested is 


$$
x+K N(x)=0, \quad x \in D(N) \subseteq Y .
$$

Our method of attack on the above equation will be by a factorization of $K$ through an appropriate Hilbert space $H$, establishing a type of determining equation for (1) in $H$, and then relating a Galerkin scheme for (1) to a Galerkin scheme for the determining equation. To this end we make the following hypotheses.

Assumptions. There exists a Hilbert space $H$, and linear mapppings $A$ : $\operatorname{Dom}(A) \subseteq X \rightarrow H, C: H \rightarrow Y$ and $D: H \rightarrow H$ such that:

(A.1) $D: H \rightarrow H$ is one-to-one and onto.

(A.2) $\operatorname{Dom}(N) \supseteq \operatorname{Range}(C), \operatorname{Dom}(A) \supseteq \operatorname{Range}(N)$ and $C D A=K$ on Range $(N)$.

(A.3) $\langle A(x), h\rangle=[x, C(h)]$, for $x \in \operatorname{Dom}(A), h \in H$.

Proposition 1.1. Under the above assumptions (A.1) and (A.2) the mapping $D^{-1}+A N C: H \rightarrow H$ is well defined, and, furthermore, if

$$
D^{-1}(v)+A N C(v)=0, \quad v \in H,
$$

then $u=C(v)$ is a solution of (1). Moreover, if $C$ is one-to-one on Range $(D A N)$, then whenever $u$ is a solution of (1) there exists $v$, a solution to (2), with $u=C(v)$.

Proof. (A.1) and (A.2) clearly imply that $D^{-1}+A N C$ is well defined. Let $v \in H$ be a solution to (2). Then by the linearity of $D$ we have

$$
v+D A N C(v)=0
$$

and again by the linearity of $C$, we get

$$
C(v)+C D A N C(v)=0 .
$$

But $N C(v) \in \operatorname{Range}(N)$ and thus (A.2) yields

$$
C(v)+K N(C(v))=0 .
$$

Now suppose that

$$
y+K N(y)=0, \quad y \in Y .
$$

Then

$$
y+C D A N(y)=0, \text { or } C(z)+C D A N C(z)=0,
$$

where $z=D A N(y)$, so that

$$
C D\left[D^{-1} z+A N C(z)\right]=0,
$$

where $D^{-1}(z)+A N C(z) \in \operatorname{Range}(A N)$, and thus we get

$$
D^{-1}(z)+A N C(z)=0 \text {. Q.E.D. }
$$

We now wish to establish a Galerkin scheme for (1), together with a companion scheme for (2). For this construction, assume that $\left\{X_{n}\right\}$ is a 
sequence of finite-dimensional subspaces of $\operatorname{Dom}(A)$, and for each positive integer $n$, let $Y_{n}=C D^{*} A\left(X_{n}\right)$ and $H_{n}=D^{*} A\left(X_{n}\right)$; so $\left\{Y_{n}\right\}$ is a sequence of finite-dimensional subspaces of $Y$ and $\left\{H_{n}\right\}$ is a sequence of finite-dimensional subspaces of $\boldsymbol{H}$. Consider the following Galerkin approximating equations for (1) and (2), respectively:

(G.1) Find $y_{n} \in Y_{n}$ such that $\left[x, y_{n}+K N\left(y_{n}\right)\right]=0$, for all $x \in X_{n}$, and

(G.2) Find $h_{n} \in H_{n}$ such that $\left\langle u, D^{-1}\left(h_{n}\right)+A N C\left(h_{n}\right)\right\rangle=0$, for all $u \in$ $H_{n}$.

Proposition 1.2. Under assumptions (A.1), (A.2) and (A.3), $y_{n} \in Y_{n}$ is a solution to (G.1) if and only if there exists $h_{n} \in H_{n}$ such that $y_{n}=C\left(h_{n}\right)$ and $h_{n}$ is a solution to (G.2).

Proof. Let $h_{n} \in H_{n}$ be such that $\left\langle u, D^{-1}\left(h_{n}\right)+A N C\left(h_{n}\right)\right\rangle=0$, for all $u \in H_{n}$. Thus,

$$
\left\langle D^{*} A(x), D^{-1}\left(h_{n}\right)+A N C\left(h_{n}\right)\right\rangle=0, \text { for all } x \in X_{n},
$$

and, consequently,

$$
\left\langle A(x), h_{n}+\operatorname{DANC}\left(h_{n}\right)\right\rangle=0, \text { for all } x \in X_{n},
$$

so that using (A.3) we obtain

or

$$
\left[x, C\left(h_{n}\right)+C D A N C\left(h_{n}\right)\right]=0, \text { for all } x \in H_{n},
$$

$$
\left[x, y_{n}+K N\left(y_{n}\right)\right]=0, \text { for all } x \in H_{n},
$$

where $y_{n}=C\left(h_{n}\right) \in Y_{n}$.

On the other hand, if $y_{n} \in Y_{n}$ and $\left[x, y_{n}+K N\left(y_{n}\right)\right]=0$, for all $x \in X_{n}$, then $y_{n}=C D^{*} A\left(x_{n}\right)$, for some $x_{n} \in X_{n}$, and a computation similar to the above shows that if $h_{n}=D^{*} A\left(x_{n}\right)$, then $h_{n}$ satisfies $\left\langle u, D^{-1}\left(h_{n}\right)+A N C\left(h_{n}\right)\right\rangle$ $=0$, for all $u \in H_{n}$. Q.E.D.

2. In this section we wish to establish a general result relating to the compactness of sequences of solutions of Galerkin equations. In special cases this will allow one to conclude that the involved mapping is $A$-proper, but we emphasize that we are not making in this section any assumptions of separability on the space, so that one cannot expect to obtain solvability of the infinite-dimensional equation based upon taking limits of a sequence of solutions to finite-dimensional equations.

Let $W$ be a Banach space, with $W^{*}$ its dual space, and for $w \in W, f \in W^{*}$ let $(f, w)$ denote the evaluation of $f$ at $w$. Recall that a mapping $T: W \rightarrow W^{*}$ is called firmly monotone provided that there exists a function $\alpha:[0, \infty) \rightarrow$ $[0, \infty)$ such that $\left\{\alpha\left(r_{n}\right)\right\} \rightarrow 0$ implies $\left\{r_{n}\right\} \rightarrow 0$ and such that

$$
(T(w)-T(v), w-v) \geqslant \alpha(\|w-v\|), \quad \text { for all } w, v \in W .
$$

A mapping $T: W \rightarrow W^{*}$ is called monotone if 


$$
(T(w)-T(v), w-v) \geqslant 0, \quad \text { for all } w, v \in W .
$$

We will need the following lemma [4], whose proof we include for completeness.

LEMMA 2.1. Let $T: W \rightarrow W^{*}$ be monotone. If $\left\{w_{n}\right\} \subseteq W$ is such that $\left\{w_{n}\right\}$ and $\left\{\left(T\left(w_{n}\right), w_{n}\right)\right\}$ are bounded, then $\left\{T\left(w_{n}\right)\right\}$ is bounded.

Proof. By the Uniform Boundedness Principle it suffices to show that for each $v \in W,\left\{\left|\left(T\left(w_{n}\right), v\right)\right|\right\}$ is bounded, and clearly to show this it suffices to show that for each $v \in W$ there is a constant $C_{v}$ such that

$$
\left(T\left(w_{n}\right), v\right) \leqslant C_{v}, \quad \text { for all } n \in \mathbf{N} .
$$

Let $v \in W$. Then

$$
\left(T\left(w_{n}\right)-T(v), w_{n}-v\right) \geqslant 0,
$$

and also

$$
\left(T\left(w_{n}\right), w_{n}\right)-\left(T\left(w_{n}\right), v\right)-\left(T(v), w_{n}\right)+(T(v), v) \geqslant 0 .
$$

Letting

$$
C_{v}=\sup _{n}\left\{\left(T\left(w_{n}\right), w_{n}\right)-\left(T(v), w_{n}\right)+(T(v), v)\right\},
$$

we see that $C_{v}<\infty$ and $\left(T\left(w_{n}\right), v\right) \leqslant C_{v}$ for all $n \in \mathbf{N}$. Q.E.D.

Before proceeding to the main result of this section, recall that a mapping $C: W \rightarrow W^{*}$ is called compact if it is continuous and $C(D)$ is relatively compact whenever $D \subseteq W$ is bounded. Also, if $M \subseteq W$ and $w \in W$ we let $d(w, M)=\inf \{\|w-z\| \mid z \in M\}$, and the symbol $w_{n} \rightarrow w_{0}$ will denote the weak convergence of $\left\{w_{n}\right\}$ to $w_{0}$.

THEOREM 2.1. Let $W$ be a reflexive Banach space, with $T: W \rightarrow W^{*}$ firmly monotone and $C: W \rightarrow W^{*}$ compact. For each $n \in \mathbf{N}$ let $W_{n}$ be a subspace of $W$ and let $P_{n}$ be a projection of $W$ onto $W_{n}$. Let $Z=\left\{w \in W \mid d\left(w, W_{n}\right) \rightarrow 0\right.$ as $n \rightarrow \infty\}$. Then whenever $\left\{w_{n}\right\}$ is a bounded sequence such that $w_{n} \in W_{n} \cap$ $Z$ for each $n \in \mathbf{N}$ and

$$
\left\|P_{n}^{*} T\left(w_{n}\right)+P_{n}^{*} C\left(w_{n}\right)-P_{n}^{*}(g)\right\| \rightarrow 0 \text { for some } g \in X^{*},
$$

there exists a subsequence of $\left\{w_{n}\right\}$ which converges strongly.

Proof. We will first show that $\left\{T\left(w_{n}\right)\right\}$ is bounded, and to do so it suffices, using Lemma 2.1, to show that $\left(T\left(w_{n}\right), w_{n}\right)$ is bounded. Now, for each $n$,

$$
\begin{aligned}
\left(T\left(w_{n}\right), w_{n}\right) & =\left(T\left(w_{n}\right), P_{n}\left(w_{n}\right)\right)=\left(P_{n}^{*} T\left(w_{n}\right), w_{n}\right) \\
& =\left(R_{n}, w_{n}\right)+\left(P_{n}^{*} g-P_{n}^{*} C\left(w_{n}\right), w_{n}\right),
\end{aligned}
$$

where $R_{n}=P_{n}^{*} T\left(w_{n}\right)+P_{n}^{*} C\left(w_{n}\right)-P_{n}^{*} g$.

But $\left\{\left(R_{n}, w_{n}\right)\right\} \rightarrow 0$, since $\left\|R_{n}\right\| \rightarrow 0$ and $\left\{w_{n}\right\}$ is bounded, and 


$$
\left(P_{n}^{*}(g)-P_{n}^{*} C\left(w_{n}\right), w_{n}\right)=\left(g-C\left(w_{n}\right), w_{n}\right),
$$

so that since $C$ is compact, $\left\{C\left(w_{n}\right)\right\}$ is bounded, and the conclusion follows.

The reflexivity of $W$ implies that we can choose a subsequence of $\left\{w_{n}\right\}$ which converges weakly to $w_{0}$, and $w_{0} \in Z$ since $Z$ is a strongly closed subspace and hence weakly closed. Furthermore, using the compactness of $C$ we may choose a further subsequence of $\left\{w_{n}\right\}$ whose image under $C$ converges strongly to $f$. Simply for convenience of notation we will suppose $\left\{w_{n}\right\} \rightarrow w_{0}$ and $\left\{C\left(w_{n}\right)\right\} \rightarrow f$.

Now

$$
\begin{aligned}
&\left(T\left(w_{n}\right)-T\left(w_{0}\right), w_{n}-w_{0}\right) \\
&=\left((T+C)\left(w_{n}\right)-(T+C)\left(w_{0}\right), w_{n}-w_{0}\right) \\
&-\left(C\left(w_{n}\right)-C\left(w_{0}\right), w_{n}-w_{0}\right) \\
&=\left((T+C)\left(w_{n}\right), w_{n}-w_{0}\right)+a_{n}+b_{n},
\end{aligned}
$$

where

$$
\begin{aligned}
& a_{n}=-\left((T \dot{+} C)\left(w_{0}\right), w_{n}-w_{0}\right) \text { and } \\
& b_{n}=-\left(C\left(w_{n}\right)-C\left(w_{0}\right), w_{n}-w_{0}\right) .
\end{aligned}
$$

Now $w_{0} \in Z$ and so we may choose $\left\{v_{n}\right\}$ with $v_{n} \in W_{n}$ for each $n$, and $d\left(w_{0}, v_{n}\right) \rightarrow 0$ as $n \rightarrow \infty$. Consequently,

$$
\begin{aligned}
((T+C) & \left.\left(w_{n}\right), w_{n}-w_{0}\right) \\
& =\left((T+C)\left(w_{n}\right), w_{n}-v_{n}\right)+\left((T+C)\left(w_{n}\right), v_{n}-w_{0}\right) \\
& =\left(P_{n}^{*}(T+C)\left(w_{n}\right), w_{n}-v_{n}\right)+c_{n},
\end{aligned}
$$

where $c_{n}=\left((T+C)\left(w_{n}\right), v_{n}-w_{0}\right)$.

We finally have, for each $n \in \mathbf{N}$,

$$
\begin{aligned}
\left(T\left(w_{n}\right)-T\left(w_{0}\right), w_{n}-w_{0}\right)= & \left(P_{n}^{*}(T+C)\left(w_{n}\right), w_{n}-v_{n}\right)+a_{n}+b_{n}+c_{n} \\
= & \left(P_{n}^{*}(T+C)\left(w_{n}\right)-P_{n}^{*}(g), w_{n}-v_{n}\right) \\
& +\left(g, w_{n}-v_{n}\right)+a_{n}+b_{n}+c_{n} .
\end{aligned}
$$

But $\left\{w_{n}-v_{n}\right\} \rightarrow 0$ and, since $\left\{P_{n}^{*}(T+C)\left(w_{n}\right)-P_{n}^{*}(g)\right\} \rightarrow 0$, we see that the first two terms of the above go to 0 as $n \rightarrow \infty$. Also, $\left\{a_{n}+b_{n}\right\} \rightarrow 0$ since $\left\{C\left(w_{n}\right)\right\} \rightarrow f$, and $\left\{c_{n}\right\} \rightarrow 0$ since $\left\{(T+C)\left(w_{n}\right)\right\}$ is bounded and $\left\{v_{n}-w_{0}\right\}$ $\rightarrow 0$.

The firm monotonicity of $T$ now implies that $w_{n}$ converges to $w_{0}$, which, keeping in mind our notational convention, means the original sequence has a strongly convergent subsequence. Q.E.D.

COROLLARY 2.1. Let the hypotheses of the previous theorem be satisfied and, 
in addition, assume $W_{n+1} \supseteq W_{n}$ for each $n \in \mathbf{N}$. Then, whenever $\left\{w_{n}\right\}$ is a bounded sequence such that $w_{n} \in W_{n}$ for each $n \in \mathbf{N}$, and

$$
\left\|P_{n}^{*}(T+C)\left(w_{n}\right)-P_{n}^{*}(g)\right\| \rightarrow 0 \text { for some } g \in X^{*},
$$

there exists a subsequence of $\left\{w_{n}\right\}$ which converges strongly.

Proof. The fact that $W_{n+1} \supseteq W_{n}$ implies that $Z={\overline{U_{n=1}^{\infty}}}_{n}$, so that $W_{n}=Z \cap W_{n}$ for each $n$. Q.E.D.

We recall that when the above sequences $\left\{W_{n}\right\}$ and $\left\{P_{n}\right\}$ have the property that $\left\{P_{n}(w)\right\} \rightarrow w$ for each $w \in W$, then the pair $\left\langle\left\{W_{n}\right\},\left\{P_{n}\right\}\right\rangle$ is said to be a projectionally complete scheme for $W$. In case one has only $\left\{d\left(w, W_{n}\right)\right\} \rightarrow 0$ for all $w \in W$, the sequence $\left\{W_{n}\right\}$ is said to be complete in $W$. In this case $Z=W$ and so $W_{n}=W_{n} \cap Z$. For later reference we record

COROLlaRY 2.2. Let the hypotheses of Theorem 2.1 hold, and, in addition, assume that $\left\{W_{n}\right\}$ is complete for $W$. Then, whenever $\left\{w_{n}\right\}$ is a bounded sequence with $w_{n} \in W_{n}$ for each $n \in \mathbf{N}$ and

$$
\left\|P_{n}^{*}(T+C)\left(w_{n}\right)-P_{n}^{*}(g)\right\| \rightarrow 0 \text { for some } g \in W^{*} \text {, }
$$

there exists a subsequence of $\left\{w_{n}\right\}$ which converges strongly.

3. In this section we will show that one can obtain solutions of (1) as limits of subsequences of solutions to (G.1). Our approach will be to use the results of $\$ 1$ to formulate (2) and (G.2), and then to use the topological degree of Brouwer to obtain solutions of (G.2) and the results of $\$ 2$ to extract strongly convergent subsequences.

Recall that a mapping $T: W \rightarrow W^{*}$ is called demicontinuous provided that $T$ is continuous from $W$, when equipped with the strong topology into $W^{*}$, where $W^{*}$ is considered equipped with the weak topology.

THEOREM 3.1. Suppose that (A.1)-(A.3) of $\$ 1$ still hold. Furthermore suppose:

(A.4) $C$ and $A$ are bounded.

(A.5) There exists a constant $c_{1}>0$ such that

$$
\left\langle D^{-1}(h), h\right\rangle \geqslant c_{1}\|h\|^{2}, \text { for all } h \in H \text {. }
$$

(A.6) $N=N_{1}+N_{2}$, where $N_{2}$ : $\operatorname{Dom}\left(N_{2}\right) \subseteq Y \rightarrow X$ is compact, $N_{1}$ is demicontinuous and satisfies

$$
\left[N_{1}(u)-N_{1}(v), u-v\right] \geqslant-c_{2}\|u-v\|^{2}, \text { for } u, v \in \operatorname{Dom}\left(N_{2}\right),
$$

and there exists a constant $c_{3}>0$ and a function $\varphi:[0, \infty) \rightarrow[0, \infty)$ such that

$$
\left[N_{2}(u), u\right] \geqslant-c_{3}\|u\|^{2}-\varphi(\|u\|), \text { for } u \in \operatorname{Dom}\left(N_{2}\right) \text {, }
$$

where $\varphi(r)=o\left(r^{2}\right)$ as $r \rightarrow \infty, \varphi([0, a])$ is bounded for each $0<a<\infty$ and $c_{1}-\left(c_{2}+c_{3}\right)\|C\|^{2}>0$.

(A.7) If $n \in \mathbf{N}$ and $h \in H_{n}$ then $\lim _{m \rightarrow+\infty} d\left(h, H_{m}\right)=0$. 
Then there exists a bounded sequence $\left\{y_{n}\right\}$ with each $y_{n} \in Y_{n}$ a solution to (G.1). Moreover, if $\left\{y_{n}^{\prime}\right\}$ is any bounded sequence such that each $y_{n}^{\prime} \in Y_{n}$ is a solution to (G.1), then a subsequence of $\left\{y_{n}^{\prime}\right\}$ converges strongly in $Y$.

Proof. We will first prove the existence of a bounded sequence $\left\{y_{n}\right\}$, with each $y_{n} \in Y_{n}$ a solution of (G.1). From Proposition 1.2 we see that it suffices to find a bounded sequence $\left\{h_{n}\right\}$, with each $h_{n} \in H_{n}$ a solution of (G.2), for then, since $C$ is bounded, we may let $y_{n}=C\left(h_{n}\right)$ to get the desired sequence.

Define $T_{n}: H_{n} \rightarrow H_{n}$ by $T_{n}=\left.P_{n} \circ\left(D^{-1}+A N C\right)\right|_{H_{n}}$, where $P_{n}$ is the orthogonal projection of $H$ onto $H_{n}$. Then $T_{n}=T_{n}^{\prime}+T_{n}^{\prime \prime}$, where

$$
\begin{aligned}
& T_{n}^{\prime}=\left.P_{n} \circ\left(D^{-1}+A N_{1} C\right)\right|_{H_{n}}, \quad \text { and } \\
& T_{n}^{\prime \prime}=\left.P_{n} \circ\left(D^{-1}+A N_{2} C\right)\right|_{H_{n}} .
\end{aligned}
$$

Now $T_{n}^{\prime}$ is demicontinuous, and, since $H_{n}$ is finite-dimensional, it is thus continuous. Since $T_{n}^{\prime \prime}$ is continuous, it follows that $T_{n}$ is continuous. Now we claim that there exists an $R>0$ such that for any $n \in \mathbf{N}$,

$$
\left\langle T_{n}(h), h\right\rangle>0 \text { if } h \in H_{n} \text { and }\|h\| \geqslant R .
$$

Indeed, we have, for $u, v \in H_{n}$,

$$
\begin{aligned}
\left\langle T_{n}(u)-T_{n}(v), u-v\right\rangle \geqslant & \left(c_{1}-c_{2}\|C\|^{2}\right)\left(\|u-v\|^{2}\right) \\
& +\left\langle A N_{2} C(u)-A N_{2} C(v), u-v\right\rangle,
\end{aligned}
$$

so that letting $v=0$, we get

$$
\langle T(u)-T(0), u\rangle \geqslant\left(c_{1}-c_{2}\|C\|^{2}\right)\|u\|^{2}+\left\langle A N_{2} C(u)-A N_{2}(0), u\right\rangle .
$$

Therefore,

$$
\begin{aligned}
\left\langle T_{n}(u), u\right\rangle \geqslant & \left(c_{1}-c_{2}\|C\|^{2}\right)\|u\|^{2}-\left\|T_{n}(0)\right\|\|u\| \\
& -\left\|A N_{2}(0)\right\|\|u\|-c_{3}\|C(u)\|^{2}+\varphi(\|C(u)\|) \\
\geqslant & \left\{c_{1}-\left(c_{2}+c_{3}\right)\|C\|^{2}\right\}\|u\|^{2}-\left\|T_{n}(0)\right\|\|u\| \\
& +\left\|A N_{2}(0)\right\|\|u\|+\varphi(\|C(u)\|) .
\end{aligned}
$$

From this it is clear that $\left\langle T_{n}(h), h\right\rangle>0$ for $h$ sufficiently large and $h \in H_{n}$ provided we can show that the $\varphi(\|C(h)\|)\left(\|h\|^{2}\right)^{-1} \rightarrow 0$ as $\|h\| \rightarrow+\infty$. If this were not the case, then we could choose a sequence $\left\{h_{n}\right\}$ in $H$ and $\beta>0$ such that $\varphi\left(\left\|C\left(h_{n}\right)\right\|\right)\left(\left\|h_{n}\right\|^{2}\right)^{-1} \geqslant \beta$ for all $n$, and $\left\{\left\|h_{n}\right\|\right\} \rightarrow+\infty$. This clearly implies $\left\{\left\|C\left(h_{n}\right)\right\|\right\} \rightarrow+\infty$ and since

$$
\varphi\left(\left\|C\left(h_{n}\right)\right\|\right)\left(\left\|h_{n}\right\|^{2}\right)^{-1} \leqslant \varphi\left(\left\|C\left(h_{n}\right)\right\|\right) \cdot\left(\left\|C\left(h_{n}\right)\right\|^{2}\right)^{-1} \cdot\|C\|^{2},
$$

we contradict the assumption that $\varphi(r)=o\left(r^{2}\right)$ as $r \rightarrow+\infty$.

The above argument allows us to choose $R>0$ such that 


$$
\left\langle T_{n}(h), h\right\rangle>0 \text { for }\|h\| \geqslant R, h \in H_{n}, n \in \mathbf{N} .
$$

A familiar homotopy argument now yields the fact that the Brouwer degree of $T_{n}$ on $\bar{B}(0, R) \cap H_{n}$ with respect to 0 is nonzero, and hence there exists $h_{n} \in \bar{B}(0, R) \cup H_{n}$ with $T_{n}\left(h_{n}\right)=0$.

It remains to show that if $\left\{y_{n}^{\prime}\right\}$ is a bounded sequence, with each $y_{n}^{\prime}$ a solution of (G.1), then $\left\{y_{n}^{\prime}\right\}$ has a convergent subsequence; to this end, we will first show that $T=D^{-1}+A N C: H \rightarrow H$ is a compact perturbation of a firmly monotone mapping. Now $T=T_{1}+T_{2}$ where $T_{1}=D^{-1}+A N_{1} C$ and $T_{2}=A N_{2} C$, and so, since $A$ and $C$ are bounded linear mappings and $N_{2}$ is compact we see that $T_{2}$ is compact, and it suffices to show that $T_{1}$ is firmly monotone. Well, for $u, v \in H$,

$$
\begin{aligned}
\left\langle T_{1}(u)-T_{2}(v), u-v\right\rangle & =\left\langle D^{-1}(u-v), u-v\right\rangle \\
& +\left\langle A N_{1} C(u)-A N_{1} C(v), u-v\right\rangle \\
\geqslant & c_{1}\|u-v\|^{2}+\left[N_{1} C(u)-N_{1} C(v), C(u)-C(v)\right] \\
\geqslant & c_{1}\|u-v\|^{2}-c_{2}\|C(u)-C(v)\|^{2} \\
\geqslant & \left(c_{1}-c_{2}\|C\|^{2}\right)\|u-v\|^{2} \\
& =\gamma\|u-v\|^{2}, \text { where } \gamma>0 .
\end{aligned}
$$

Now, letting $P_{n}$ be the orthogonal projection of $H$ onto $H_{n}$, we see that $h \in H_{n}$ satisfies (G.2) if and only if $P_{n}^{*}\left(D^{-1}+A N C\right)(h)=0$. Hence, if $\left\{h_{n}\right\}_{n>n_{0}}$ is such that $\left\{h_{n}\right\}$ is bounded and each $h_{n}$ is a solution to (G.2), then

$$
\left\{P_{n}^{*}\left(D^{-1}+A N C\right)\left(h_{n}\right)-P_{n}^{*}(0)\right\} \rightarrow 0,
$$

and, consequently, we may invoke Theorem 2.1 to conclude that $\left\{h_{n}\right\}$ has a strongly convergent subsequence. Now if $\left\{y_{n}^{\prime}\right\}$ is a bounded sequence of solutions of (G.1), then Proposition 1.2 guarantees that $y_{n}^{\prime}=C\left(h_{n}\right)$, where $h_{n}$ is a solution of (G.2), for each $n$. Thus $\left\langle T_{n}\left(h_{n}\right), h_{n}\right\rangle=0$, and from the a priori estimate of the first part of the proof it follows that $\left\|h_{n}\right\| \leqslant R$ for each $n$. Thus $\left\{h_{n}\right\}$ has a strongly convergent subsequence, and so, since $C$ is bounded, $\left\{y_{n}^{\prime}\right\}$ has a strongly convergent subsequence. Q.E.D.

The following simple result gives useful conditions under which hypothesis (A.7) may be verified.

PROPOSITION 3.1. Using the notation of $\$ 1$, assume that either

(A.7)' $X_{n} \subseteq X_{n+1}$ for each $n$, or

(A.7)" $d\left(x, X_{n}\right) \rightarrow 0$ for all $x \in X$ (i.e. $\left\{X_{n}\right\}$ is complete for $\left.X\right)$.

Then hypothesis (A.7) holds. 
Proof. If (A.7)' holds then $H_{n} \subseteq H_{n+1}$ for each $n$ so that $d\left(h, H_{m}\right)=0$ for $m \geqslant n$ and $h \in H_{m}$.

In case (A.7)" holds, then the fact that $\lim _{m \rightarrow \infty} d\left(x, X_{m}\right)=0$ for each $x \in X_{n}$, together with $D^{*}$ and $A$ being bounded, implies

$$
\lim _{m \rightarrow \infty} d\left(D^{*} A(x), D^{*} A\left(X_{m}\right)\right)=0,
$$

so that $\lim _{m \rightarrow \infty} d\left(h, H_{m}\right)=0$, for $h \in H_{n}$. Q.E.D.

So far, of course, we have not imposed any denseness conditions with respect to $\left\{X_{n}\right\}$ and $X \times Y$, and so we cannot expect to be able to conclude very much about the limit of the subsequence whose existence is guaranteed by Theorem 3.1. The next proposition deals with this point.

Proposition 3.2. In addition to (A.1)-(A.3) of \$1, suppose the following:

(A.8) If $y \in Y$ and $[x, y]=0$ for all $x \in \cup_{n=1}^{\infty} X_{n}$, then $y=0$.

Moreover, suppose that $N$ is demicontinuous and $K$ is bounded. Let $\left\{y_{n_{k}}\right\}$ be a bounded sequence with $y_{n_{k}} \in Y_{n_{k}}$ for each $k$ and $\left[x, y_{n_{k}}+K N\left(y_{n_{k}}\right)\right]=0$, for all $x \in X_{n_{k}}$, and suppose $\left\{y_{n_{k}}\right\}$ converges to $y_{0} \in \operatorname{Dom}(N)$. Then, if either (A.7)' or (A.7)" holds, $y_{0}+K N\left(y_{0}\right)=0$.

Proof. First suppose (A.7)' holds. Fix $j \in \mathbf{N}$ and let $x \in X_{j}$. Then $\left[x, y_{n_{k}}+\right.$ $\left.K N\left(y_{n_{k}}\right)\right]=0$, if $n_{k}>j$, and thus letting $k \rightarrow \infty$ and using the demicontinuity of $N$ and the boundedness of $K$, we get $\left[x, y_{0}+K N\left(y_{0}\right)\right]=0$.

By our arbitrary choice of $j \in \mathbf{N}$ and hypothesis (A.8) we conclude that $y_{0}+K N\left(y_{0}\right)=0$.

Now suppose (A.7)" holds. Let $x \in X$. Then we may choose $\left\{x_{n_{k}}\right\}$ such that $\left\{x_{n_{k}}\right\} \rightarrow x$ and $x_{n_{k}} \in X_{n_{k}}$ for each $k$. The boundedness of $K$ and the demicontinuity of $N$ imply that $y_{n_{k}}+K N\left(y_{n_{k}}\right)-y_{0}+K N\left(y_{0}\right)$, and since

$$
\left[x_{n_{k}}, y_{n_{k}}+K N\left(y_{n_{k}}\right)\right]=0 \text {, for each } k \text {, }
$$

the continuity of the bilinear form [, ], guarantees that

$$
\left[x, y_{0}+K N\left(y_{0}\right)\right]=0 \text {. }
$$

Again, (A.8) implies that $y_{0}+K N\left(y_{0}\right)=0$. Q.E.D.

Our final assumption will be

(A.9) $\overline{\cup_{n=1}^{\infty} Y_{n}} \supseteq \operatorname{Dom}(N)$.

We may now state our general constructive existence result for the abstract Hammerstein equation.

TheOREM 3.2. Suppose that (A.1)-(A.9) hold, together with (A.7)' or (A.7)". Then there exists a bounded sequence $\left\{y_{n}\right\}$, such that each $y_{n} \in Y_{n}$ is a solution to (G.1). Moreover, if $\left\{y_{n}^{\prime}\right\}$ is any bounded sequence, with each $y_{n}^{\prime} \in Y_{n}$ a solution of (G.1), then $\left\{y_{n}^{\prime}\right\}$ has a subsequence which converges strongly to a solution of (1); if the solution to (1) is unique, the whole sequence $\left\{y_{n}^{\prime}\right\}$ converges to this solution. 
4. Angle bounded mappings. Let $Y \subseteq X^{*}$, and let [ , ] be the bilinear form induced on $X \times Y$ by evaluation, i.e. $[x, y]=y(x)$, for $x \in X, y \in Y \subseteq X^{*}$.

Following Amann [2], [3] we call a bounded linear mapping $K: X \rightarrow Y$ angle-bounded provided that it is monotone (i.e. $(x, K(x)) \geqslant 0$ for each $x \in X)$ and there exists a constant $a>0$ such that

$$
|(x, K(y))-(y, K(x))| \leqslant 2 a(x, K(x))^{1 / 2}(y, K(y))^{1 / 2},
$$

for all $x, y \in X$. Clearly, when $a=0$ we are in the case of a positive symmetric mapping, and then there exists a square-root for $K$ and, consequently, a suitable splitting for $K$. The following result of Browder and Gupta [7] (see also Amman [2], [3]) produces a similar splitting in the angle-bounded case.

Proposition 4.1. Let $X$ be a banach space with $Y$ a closed subspace of $X^{*}$. Let $K: X \rightarrow Y$ be angle-bounded. Then there exists a Hilbert space $H, a$ bounded linear mapping $A: X \rightarrow H$, and a skew-symmetric bounded linear map $B: H \rightarrow H$ such that

(i) $K=A^{*}(I+B) A$,

and, furthermore, $\|A\|^{2} \leqslant\|K\|$ and $\|B\| \leqslant a$, where $a$ is the constant of angle-boundedness. Finally, the following inequality is satisfied.

(ii) $\left\langle(I+B)^{-1}(h), h\right\rangle \geqslant\left(1+a^{2}\right)^{-1}\|h\|^{2}$.

REMARK. The assumption of the boundedness of $K$ is redundant, but it seems worthwhile to note explicitly this property.

The above proposition allows us to put the Hammerstein equations whose linear part is angle-bounded into our general framework. In the terminology of $\S 1, A^{*}$ plays the role of $C, I+B$ plays the role of $D$, and the fact that $C=A^{*}$ guarantees the compatibility of the inner product on $H$ and the bilinear form on $X \times Y$. Furthermore, when $K$ maps $X$ into $X^{*}$, then one lets $X^{*}=Y$, and when $K: X^{*} \rightarrow X$, then one lets $X^{*}$ play the role of $X$ and lets $X$ play the role of $Y$, viewing $X$ as being embedded in the usual manner as a closed subspace of $\left(X^{*}\right)^{*}$. Of course, when $X$ is reflexive there is no distinction between these two cases; however, in the nonreflexive case, although the general reformulation results of $\$ 1$ carry over, there has to be a distinction made in view of the fact that the separability of $X$ does not imply the separability of $X^{*}$, and this situation, in its turn, focuses rather precisely upon the distinction between (A.7)' and (A.7)" of $\$ 3$.

REMARK 4.1. The idea of the proof of Proposition 4.1 is as follows: $\left(K+K^{*}\right) / 2$ is a nonnegative symmetric mapping of $X$ into $X^{*}$ and ( $K-$ $\left.K^{*}\right) / 2$ is an antisymmetric mapping of $X$ into $X^{*}$, and, of course, $K=(K+$ $\left.K^{*}\right) / 2+\left(K-K^{*}\right) / 2$.

Now, by factoring out the space $N=\{x \mid(x, K(x))=0\}$, introducing the 
inner-product $\langle\bar{x}, \bar{y}\rangle=(x, K(y))$ for $\bar{x}=x+N, \bar{y}=y+N$ in the factor space, completing the factor space to a Hilbert space $H$, and letting $S$ denote the embedding of $X$ into $H$, one gets $\left(K+K^{*}\right) / 2=S^{*} S$ and $\left(K-K^{*}\right) / 2=$ $S^{*} B S$, where $B$ is a bounded antisymmetric mapping. We recall this construction to point out that the mapping $S^{*}(I-B) S=K^{*}$, so that the finite-dimensional space $Y_{n}=K^{*}\left(X_{n}\right)$, and, consequently, in the notation we have been using,

$$
\left[x, y_{n}+K N\left(y_{n}\right)\right]=0, \quad y_{n} \in Y_{n}, \text { for all } x \text { in } X_{n},
$$

is equivalent to

$$
\left(x, K^{*}\left(z_{n}\right)+K N K^{*}\left(z_{n}\right)\right)=0, \quad z_{n} \in X_{n}, \quad \text { for all } x \in X_{n} .
$$

Thus we see that in this case our Galerkin scheme is simply the natural Galerkin scheme one would consider on transforming (1) into

$$
\left(K^{*}+K N K^{*}\right)(z)=0, \quad z \in X .
$$

THEOREM 4.1. Let $X$ be a separable Banach space with $\left\{X_{n}\right\}$ a sequence of finite-dimensional subspaces of $X$ such that $\lim _{n \rightarrow \infty} d\left(x, X_{n}\right)=0$ for each $x \in X$. Suppose $K: X \rightarrow Y \subseteq X^{*}$ is angle-bounded with constant of angleboundedness $a$, where $Y$ is a closed subspace of $X^{*}$. Let $N: Y \rightarrow X$ be such that $N=N_{1}+N_{2}$, where $N_{2}$ is compact, $N_{1}$ is demicontinuous and there exist constants $c_{1}>0$ and $c_{2}>0$ and a function $\varphi:[0, \infty) \rightarrow[0, \infty)$ such that:

(i) $c_{1}+c_{2} \leqslant\|K\| /\left(1+a^{2}\right)$.

(ii) $\left(N_{1}(u)-N_{1}(v), u-v\right) \geqslant-c_{1}\|u-v\|^{2}$, for $u, v \in Y^{*}$.

(iii) $\left(N_{2}(u), u\right) \geqslant-c_{2}\|u\|^{2}-\varphi(\|u\|)$, for $u \in Y^{*}$ where $\varphi(r)=o\left(r^{2}\right)$ as $r \rightarrow+\infty$, and $\varphi([0, a])$ is bounded for each $0<a<\infty$.

Then there exists a bounded sequence $\left\{y_{n}\right\}$ such that for each $n, y_{n} \in K^{*}\left(X_{n}\right)$ and

$$
\left(x, y_{n}+K N\left(y_{n}\right)\right)=0, \text { for all } x \in X_{n} .
$$

Furthermore, if $\left\{y_{n}^{\prime}\right\}$ is any bounded sequence having the above property then $\left\{y_{n}^{\prime}\right\}$ has a subsequence which converges to a solution of (1).

PRoof. Proposition 4.1 guarantees that the algebraic results of $\$ 1$ hold, and Remark 4.1 identifies $Y_{n}$ as $K^{*}\left(X_{n}\right)$. Again the estimates from Proposition 4.1 together with the estimates imposed on $N$ allows us to invoke Theorem 3.2 in the case that (A.7)" holds. Q.E.D.

REMARK. Theorem 4.1 includes Theorems 1 and 2 of Amann [2], who proved the convergence of the Galerkin method for the case when $N_{1}$ is continuous, $N_{2} \equiv 0, X_{n} \subseteq X_{n+1}$ for each $n$, and $X$ has a certain embedding property: the continuity of $N$ is essential for the type of proof used in [2].

In case the Hammerstein equation is formulated so that $K: X^{*} \rightarrow X$ and $N$ : $X \rightarrow X^{*}$, the following result holds. 
THEOREM 4.2. Let $X$ be a Banach space which has a Schauder basis $\left\{z_{n}\right\}$, with corresponding evaluation functionals $\left\{f_{n}\right\}$. For each $n \in \mathbf{N}$ let $F_{n}=$ $\operatorname{span}\left\{f_{i} \mid 1<i \leqslant n\right\}$. Suppose $K: X^{*} \rightarrow X$ is angle-bounded with constant of angle-boundedness $a$, and $N: X \rightarrow X^{*}$ is such that $N=N_{1}+N_{2}$ where $N_{1}$ and $N_{2}$ satisfy the hypotheses outlined in Theorem 4.1. Then there exists a bounded sequence $\left\{x_{n}\right\}$ with $x_{n} \in K^{*}\left(F_{n}\right)$ for each $n$ and

$$
\left(g, x_{n}+K N\left(x_{n}\right)\right)=0, \text { for all } g \in F_{n} \text {. }
$$

Furthermore, if $\left\{x_{n}^{\prime}\right\}$ is any bounded sequence having the above property then $\left\{x_{n}^{\prime}\right\}$ has a subsequence which converges strongly to a solution of $x+K N(x)=$ 0 .

Proof. This proof follows in the same manner as that of Theorem 4.1 except that now we use the fact that $F_{n+1} \supseteq F_{n}$ for each $n$ and the weak* denseness of $\left\{f_{n} \mid n \in \mathbf{N}\right\}$ to verify (A.7)' and (A.8). Q.E.D.

\section{Applications to ordinary and partial differential equations.}

Proposition 5.1. Let $X$ be a Hilbert space and $Y$ a Banach space, and let $\bar{K}$ : $Y \rightarrow X$ be bounded linear, one-to-one and onto. Suppose $\bar{K}=\bar{A} \circ \bar{C}$, where $\bar{A}$ and $\bar{C}$ are linear. Define $H=\bar{C}(Y)$ and on $H$ define an inner-product $\langle,>$ by

$$
\langle u, v\rangle=\langle\bar{A}(u), \bar{A}(v)\rangle_{X} .
$$

Then, if on $X \times Y$ we define a bilinear form $[x, y]=\langle x, \bar{K}(y)\rangle_{X}$, and we define $K=(\bar{K})^{-1}, A=(\bar{A})^{-1}$ and $C=(\bar{C})^{-1}$, then $H$ is a Hilbert space and $A: X \rightarrow H$ and $C: H \rightarrow Y$ are bounded mappings such that $K=C \circ A$ and

$$
\langle A(x), h\rangle=[x, C(h)], \text { for } x \in X, h \in H .
$$

Furthermore, $\|C\|=\|K\|$.

Proof. It is clear that $\langle$,$\rangle is a bilinear form on H$, so to show that it an inner-product we need only show that if $h \in H$ and $\langle h, h\rangle=0$, then $h=0$. But $\langle h, h\rangle=0$ means $\langle\bar{A}(h), \bar{A}(h)\rangle_{X}=0$, so that $\bar{A}(h)=0$; also $h=\bar{C}(y)$, so that $\bar{K}(y)=0$. Thus $y=0$, and, consequently, $h=0$. Now to prove completeness: let $\left\{h_{n}\right\} \subseteq H$ be Cauchy; then $\left\{\bar{A}\left(h_{n}\right)\right\}$ is Cauchy in $X$ and, letting $h_{n}=\bar{C}\left(y_{n}\right)$ for each $n$, we have $\left\{\bar{K}\left(y_{n}\right)\right\}$ Cauchy. Since $(\bar{K})^{-1}$ is continuous we have $\left\{y_{n}\right\}$ Cauchy, and so $\left\{y_{n}\right\}$ converges to $y_{0}$ in $Y$, with $\left\{\bar{K}\left(y_{n}\right)\right\}$ converging to $\bar{K}\left(y_{0}\right)$. This means $\left\{h_{n}\right\}$ converges to $\bar{C}\left(y_{0}\right)$ in $H$.

Now $K=C \circ A$ and noting that

$$
\langle A(x), h\rangle=\left\langle(\bar{A})^{-1}(x), h\right\rangle=\langle x, \bar{A}(h)\rangle_{X},
$$

while

$$
[x, C(h)]=\left[x,(\bar{C})^{-1}(h)\right]=\left\langle x, \bar{K} \circ(\bar{C})^{-1}(h)\right\rangle_{X}=\langle x, \bar{A}(h)\rangle_{X}
$$


we get $\langle A(x), h\rangle=[x, C(h)]$, for $x \in X, h \in H$. Finally, since $A$ is an isometry, $\|C\|=\|K\|$. Q.E.D.

We will apply the results of the previous sections to differential equations by means of Proposition 5.1; but first, we recall some notation.

If $k \in \mathbf{N}$ and $\Omega \subseteq \mathbf{R}^{k}$ is a bounded domain, then for $m \in \mathbf{N}$ we define $H^{m}(\Omega)$ to be $\left\{u \in L^{2}(\Omega) \mid D^{\alpha} u \in L^{2}(\Omega)\right.$ for $\left.|\alpha| \leqslant m\right\}$, where $\alpha=\left(\alpha_{1} \ldots \alpha_{m}\right)$ $\in \mathbf{N}^{m},|\alpha|=\sum_{i=1}^{m}\left|\alpha_{i}\right|$, and $D^{\alpha} u$ denotes the weak derivative of $u$. Then $H^{m}(\Omega)$ is a Banach space under the norm

$$
\|u\|_{H^{m}} \equiv\left\{\sum_{|\alpha|<m}\left[\left\|D^{\alpha}(u)\right\|_{L^{2}(\Omega)}\right]^{2}\right\}^{1 / 2}, \text { for } u \in H^{m}(\Omega) \text {. }
$$

By $H_{0}^{m}(\Omega)$ we shall denote the closure, in $H^{m}(\Omega)$, of the infinitely differentiable functions which are compactly supported in $\Omega$. It is known that if $m>n / 2$, and $\Omega$ satisfies the cone condition, then $H^{j+m}(\Omega)$ is compactly embedded in $H^{j}(\Omega)$ for each $j \in \mathbf{N}$ (Adams [1, Theorem 6.2]).

Now let us consider the ordinary differential equation

$$
\left\{\begin{array}{l}
u^{\prime \prime}(t)+f\left(t, u(t), u^{\prime}(t), u^{\prime \prime}(t)\right)=0, \\
u(0)=u(1)=0
\end{array}\right.
$$

The mapping $d^{2} / d t^{2}: H^{2}([0,1]) \cap H_{0}^{1}([0,1]) \rightarrow L^{2}$ is a continuous bijection, and so we may apply Proposition 5.1: let $X=L^{2}([0,1]), Y=$ $H^{2}([0,1]) \cap H_{0}^{1}([0,1])$, and $H=\left\{u^{\prime} \mid u \in Y\right\}$; then $H$ becomes a Hilbert space under the inner product

$$
\langle u, v\rangle=\int_{0}^{1} u^{\prime}(t) v^{\prime}(t) d t, \quad \text { for } u, v \in H,
$$

and on $X \times Y$ we define a bilinear form by $[u, v]=\int_{0}^{1} u(t) v^{\prime \prime}(t) d t$.

Then letting $A: X \rightarrow H, C: H \rightarrow Y$ and $K: X \rightarrow Y$ be defined by

$A(u)=v$, where $v \in H$ and $v^{\prime}=u$,

$C(u)=v$, where $v \in Y$ and $v^{\prime}=u$, and

$K(u)=v$, where $v \in Y$ and $v^{\prime \prime}=u$,

we see that $K=C \circ A, C$ and $A$ are bounded and $\langle A(x), h\rangle=[x, C(h)]$ for $x \in X, h \in H$.

Then, letting $N: H^{2} \cap H_{0}^{1} \rightarrow L^{2}$ be the Nemytsky operator generated by $f$, we consider (5.1) in the form

$$
u+K N(u)=0, \quad u \in H^{2} \cap H_{0}^{1} .
$$

Now that the form of the linear part of the equation has been specified we can proceed to form hypotheses on $N$. First we suppose that

$$
f(t, x, y, z)=g(t, x, y)+h(t, z), \text { for }(t, x, y, z) \in[0,1] \times \mathbf{R}^{3} .
$$

Now suppose that $g$ and $h$ satisfy the Carathéodory conditions: i.e., for 
each $(x, y, z) \in \mathbf{R}^{3}$ the mappings

$$
\begin{aligned}
& t \mapsto g(t, x, y) \quad \text { for } t \in[0,1], \\
& t \mapsto h(t, z) \text { for } t \in[0,1]
\end{aligned}
$$

are measurable, while for almost all $t \in[0,1]$ the mappings

$$
\begin{aligned}
(x, y) & \mapsto g(t, x, y), \quad \text { for }(x, y) \in \mathbf{R}^{2}, \\
z & \mapsto h(t, z), \quad \text { for } z \in \mathbf{R}
\end{aligned}
$$

are continuous.

The above assumptions guarantee that $N$ maps $H^{2}$ into measurable functions: the following Nemytsky growth conditions guarantee that $N$ maps $H^{2}$ into $L^{2}$ continuously:

There exist $\varphi, \psi \in L^{2}([0,1])$ and a constant $\rho$ such that

$$
\begin{aligned}
& |g(t, x, y)| \leqslant \rho\left[\varphi(t)+\sqrt{x^{2}+y^{2}}\right] \text { for }(t, x, y) \in[0,1] \times \mathbf{R}^{2}, \\
& \text { and }|h(t, z)| \leqslant \rho[\psi(t)+|z|] \text { for }(t, z) \in[0,1] \times \mathbf{R} .
\end{aligned}
$$

If we now let $N_{1}$ and $N_{2}$ denote the Nemytsky operators defined by

$$
\begin{aligned}
& N_{2}(u)(t)=g\left(t, u(t), u^{\prime}(t)\right), \quad \text { for } t \in[0,1], u \in Y, \\
& N_{1}(u)(t)=h\left(t, u^{\prime \prime}(t)\right), \quad \text { for } t \in[0,1], u \in Y,
\end{aligned}
$$

then the fact that $H^{2} \cap H_{0}^{1}$ is compactly embedded in $H^{1}([0,1])$ and $N_{2}$ : $H^{1}([0,1]) \rightarrow L^{2}([0,1])$ is continuous yield the compactness of $N_{2}: Y \rightarrow X$.

In order to apply Theorem 3.2 we finally need to impose some coercivitytype assumption on $N$ and a monotonicity-type condition on $N_{1}$ :

(5.3) There exists a positive constant $M$ such that

$$
\left(h\left(t, z_{1}\right)-h\left(t, z_{2}\right)\right) \times\left(z_{1}-z_{2}\right) \geqslant-M_{2}\left|z_{1}-z_{2}\right|^{2} \text {, }
$$

for all $\left(t, z_{1}, z_{2}\right) \in[0,1] \times \mathbf{R}^{2}$, with $\left(\rho\left(\beta^{2}-1\right)^{1 / 2}+M\right) \alpha^{2}<(1 / \beta)^{2}$, where $\alpha$ denotes the norm of the mapping in $d^{2} / d t^{2}: H^{2} \cap H_{0}^{1} \rightarrow L^{2}$ and $\beta$ denotes the norm of the inverse mapping.

THEOREM 5.1. Assume the functions $h$ and $g$ satisfy the Carathéodory condition (C), the Nemytsky condition $(\mathrm{N})$ and hypothesis (5.3). Let $\left\{Y_{n}\right\}$ be any sequence of finite-dimensional subspaces of $H^{2} \cap H_{0}^{1}([0,1])$ which have the property that if we let $X_{n}=d^{2}\left(Y_{n}\right) / d x^{2}$, for each $n$, then $\left\{X_{n}\right\}$ is complete in $L^{2}([0,1])$. Then there exists a bounded sequence $\left\{u_{n}\right\}$ such that for each $n$, $u_{n} \in Y_{n}$ and

$$
\int_{0}^{1} u \cdot\left[u_{n}^{\prime \prime}+f\left(t, u_{n}(t), u_{n}^{\prime}(t), u_{n}^{\prime \prime}(t)\right)\right] d t=0, \text { for ali } u \in X_{n} .
$$

Furthermore, if $\left\{\bar{u}_{n}\right\}$ is any bounded sequence with $\bar{u}_{n} \in Y_{n}$ and $\bar{u}_{n}$ satisfying the system (5.4) for each $n$, then $\left\{\bar{u}_{n}\right\}$ has a subsequence which converges in $H^{2}$ 
to a solution of

$$
\left\{\begin{array}{l}
u^{\prime \prime}+f\left(t, u(t), u^{\prime}(t), u^{\prime \prime}(t)\right)=0 \\
u(0)=u(1)=0
\end{array}\right.
$$

Finally, if the solution to this equation is unique, which it is when $M_{1}=M_{2}$ $=0$, then every bounded sequence of solutions of (5.4) converges strongly to a solution of (5.5).

Proof. We know that $\alpha>0$ satisfies

$$
\left\|u^{\prime \prime}\right\|_{L^{2}}^{2} \leqslant \alpha^{2}\|u\|_{H^{2}}^{2} \quad \text { for all } u \in H^{2} \cap H_{0}^{1} .
$$

Then (5.3) guarantees that

$$
\begin{aligned}
\int_{0}^{1}(h(t, & \left.\left.u^{\prime \prime}(t)\right)-h\left(t, v^{\prime \prime}(t)\right)\right)\left(u^{\prime \prime}(t)-v^{\prime \prime}(t)\right) d t \\
& \geqslant-M \int_{0}^{1}\left|u^{\prime \prime}(t)-v^{\prime \prime}(t)\right|^{2} d t \\
& \geqslant-M \alpha^{2}\|u-v\|_{H^{2}}^{2}, \quad \text { for each } u, v \in H^{2} \cap H_{0}^{1}:
\end{aligned}
$$

i.e. $\left[N_{1}(u)-N_{1}(v), u-v\right] \geqslant-M \alpha^{2}\|u-v\|^{2}$, for $u, v \in Y$. On the other hand,

$$
\left|\int_{0}^{1} g\left(t, u(t), u^{\prime}(t)\right) u^{\prime \prime}(t) d t\right| \leqslant \rho\|\varphi\|_{L^{2}}\left\|u^{\prime \prime}\right\|_{L^{2}}+\rho\left(\beta^{2}-1\right)^{1 / 2}\left\|u^{\prime \prime}\right\|_{L^{2}}^{2}
$$

so that

$$
\begin{aligned}
\int_{0}^{1} g\left(t, u(t), u^{\prime}(t)\right) u^{\prime \prime}(t) d t=\left[N_{2}(u), u\right] \\
\geqslant-\rho\left(\beta^{2}-1\right)^{1 / 2} \alpha^{2}\|u\|_{H^{2}}^{2}+\gamma\left\|u^{\prime \prime}\right\|_{H^{2 \cdot}}
\end{aligned}
$$

Now Proposition 5.1 shows that $\|C\|=\beta$, and, consequently, the conditions imposed on the constants in (A.6) reduce to the prescribed inequality in (5.3)

$$
\left(M_{1} / \sqrt{2}+M_{2}\right) \cdot \alpha^{2}>1 / \beta^{2}
$$

Most of the proof has already been completed. One observes that, in our previous terminology, $Y_{n}=K\left(X_{n}\right)=C D^{*} A\left(Z_{n}\right)$, since $D=I$, and

$$
\left[u, u_{n}+K N\left(u_{n}\right)\right]=0, \quad \text { for all } u \in X_{n},
$$

where $u_{n} \in Y_{n}$, if and only if $u_{n}$ satisfies (5.4).

The last assertion of the theorem, namely the uniqueness when $M_{1}=M_{3}$ $=0$, follows from the firm monotonicity of $I+A N C$ in this situation. Q.E.D.

REMARK. The constants $\alpha$ and $\beta$ can be given explicitly.

The biharmonic operator. Let $\Omega$ be a domain in $\mathbf{R}^{k}, 1 \leqslant k<7$, which 
satisfies the cone property, and let $Y$ be a complete subspace of $H^{4}(\Omega)$ such that $\Delta^{2}: Y \subseteq H^{4} \rightarrow L^{2}$ is a bijection. Then the equation we shall consider is

$$
\Delta^{2}(u)+N(u)=0,\left.\quad B(u)\right|_{\partial \Omega}=0,
$$

where $N(u)(x)=\psi\left(x, \Delta^{2} u(x)\right)+\varphi(x, u(x))$, and where $B$ is a boundary operator whose natural completion in the generalized functions corresponds to $Y$. We give two examples. If $B(u)=(u, \partial u / \partial \eta)$, then (5.6) is simply

$$
\Delta^{2}(u)+N(u)=0 \text { on } \Omega, \quad u=\partial u / \partial \eta=0 \text { on } \partial \Omega,
$$

and $Y=H^{4} \cap H_{0}^{2}$, while if $B(u)=(u, \Delta u)$, then (5.6) corresponds to the so-called Riquier Problem

$$
\Delta^{2}(u)+N(u)=0 \text { on } \Omega, \quad u=\Delta u=0 \text { on } \partial \Omega,
$$

and $Y=\left\{u \in H^{4} \cap H_{0}^{1} \mid \Delta u \in H_{0}^{1}\right\}$ (see Browder [5]).

According to our abstract Proposition 5.1 the inverse of the biharmonic operator acting from $L^{2}$ to $Y$ factors as follows: let $X=L^{2}$, and $H=\Delta(Y)$, consider $H$ as being a Hilbert space when equipped with the inner product

$$
\langle u, v\rangle=\int_{\Omega} \Delta u \Delta v d x,
$$

and let the operators $K, A$ and $C$ be defined as follows:

$K: X \rightarrow Y$ is defined by $K(u)=v$, where $v \in Y, \Delta^{2}(v)=u$,

$A: X \rightarrow H$ is defined by $A(u)=v$, where $v \in H, \Delta(v)=u$,

$C: H \rightarrow Y$ is defined by $C(u)=v$, where $v \in Y, \Delta(v)=u$.

Then $A, C$ and $K$ are bounded linear mappings such that $K=C \circ A$.

We may now reformulate (5.6) as

$$
u+K N(u)=0, \quad u \in Y,
$$

and impose the following hypothesis:

(5.8) $\psi: \Omega \times \mathbf{R} \rightarrow \mathbf{R}$ and $\varphi: \Omega \times \mathbf{R} \rightarrow \mathbf{R}$ satisfy the Nemytsky condition $(\mathbb{N})$ and the Carathéodory condition (C), and there exist nonnegative constants $M_{1}$ and $M_{2}$ such that

$$
\begin{gathered}
(\psi(x, z)-\psi(x, w)) \times(z-w) \geqslant-M_{1}|z-w|^{2} \text { for all } x \in \Omega, z, w \in \mathbf{R}, \\
|\varphi(x, z)| \leqslant M_{2} \text { for all } x \in \Omega, z \in \mathbf{R},
\end{gathered}
$$

and

$$
\left(M_{1}\right) \alpha^{2}<1 / \beta^{2},
$$

where $\alpha$ denotes the norm of $\Delta^{2}: Y \rightarrow L^{2}$ and $\beta$ denotes the norm of the inverse mapping.

Theorem 5.2. Assume the functions $\psi$ and $\varphi$ satisfy (5.8). Let $\left\{Y_{n}\right\}$ be a sequence of finite-dimensional subspaces of $Y \subseteq W^{4}$ such that if $\Delta^{2}\left(Y_{n}\right) \equiv X_{n}$, for each $n \in \mathbf{N}$, then $\left\{X_{n}\right\}$ is complete in $L^{2}(\Omega)$. Then there exists a bounded 
sequence $\left\{u_{n}\right\}$ such that for each $n \in \mathbf{N}, u_{n} \in Y_{n}$, and

$$
\begin{array}{r}
\int_{\Omega} u(x) \cdot\left[\Delta^{2}\left(u_{n}\right)(x)+\psi\left(x, \Delta^{2}\left(u_{n}\right)(x)\right)+\varphi\left(x, u_{n}(x)\right)\right] d x=0 \\
\text { for each } u \in X_{n} .
\end{array}
$$

Furthermore, if $\left\{\bar{u}_{n}\right\}$ is any bounded sequence with $\bar{u}_{n} \in Y_{n}$ and $\bar{u}_{n}$ satisfying system (5.9) for each $n$, then $\left\{\bar{u}_{n}\right\}$ has a subsequence which converges strongly in $W^{4}$ to a solution of

$$
\left\{\begin{array}{l}
\Delta^{2}(u)(x)+\psi\left(x, \Delta^{2}(u)(x)\right)+\varphi(x, u(x))=0, \\
u \in Y .
\end{array}\right.
$$

Finally, when the solution of the above equation is unique, which it is when $M_{1}=0$, every bounded sequence of solutions to (5.9) converges to this unique solution.

REMARK. The proof of the above theorem follows in the same manner as that of Theorem 5.1. In case $\Omega \subseteq \mathbf{R}^{k}$ and $1 \leqslant k<7$, then the nonlinearity induced by $\varphi$ can be generalized to allow dependence on higher order derivatives; specifically, $\varphi$ may depend upon derivatives, whose orders are less than or equal to $\alpha$, of the involved function $u \in W^{4} \cap W_{0}^{2}$, provided that $\alpha<4-k / 2$ (see Adams [1]).

REMARK. The existence part of the above theorem includes as a special case the results of [9], where the nonlinearity is of a less general nature; the method of proof in [9] precludes a nonlinear function of the higher order terms entering into the equation.

\section{REFERENCES}

1. R. A. Adams, Sobolev spaces, Academic Press, New York, 1975.

2. H. Amann, Ein Existenz und Eindeutigkeitssatz für die Hammersteinshe Gleichungen in Banachraümen, Math. Z. 3 (1969), 175-190.

3. _ Zum Galerkin-Vehrfahren für die Hammersteinsche Gleichung, Arch. Rational Mech. Anal. 35 (1969), 114-121.

4. H. Brezis, Equations et inéquations non-linéaires dans les espaces vectoriels en dualité, Ann. Inst. Fourier (Grenoble) 18 (1968), 115-175.

5. F. E. Browder, Functional analysis and partial differential equations. II, Math. Ann. 145 (1962), 81-226.

6. Nonlinear functional analysis and nonlinear integral equations of Hammerstein and Unysohn type, Contributions to Nonlinear Functional Analysis, Academic Press, New York, 1971.

7. F. E. Browder and C. P. Gupta, Monotone operators and nonlinear integral equations of Hammerstein type, Bull. Amer. Math. Soc. 76 (1969), 1347-1353.

8. C. L. Dolph and G. J. Minty, On nonlinear integral equations of Hammerstein type, Nonlinear Integral Equations, Univ. of Wisconsin Press, Madison, Wisconsin, 1964, pp. 99-154.

9. D. R. Dunninger and J. Locker, Monotone operators and nonlinear biharmonic boundary value problems, Pacific J. Math. 60 (1975), 39-48. 
10. W. V. Petryshyn and P. M. Fitzpatrick, New existence theorems for nonlinear equations of Hammerstein type, Trans. Amer. Math. Soc. 160 (1971), 39-63.

11. M. Vainberg, Variational methods in the study of nonlinear operators, Moscow, 1956; English transl., Holden-Day, San Francisco, Calif., 1963.

Department of Mathematics, University of Maryland, College Park, Maryland 20742

Department of Mathematics, Rutgers University, New Brunswick, New Jersey 08930 